\title{
MATHEMATICAL MODELING OF WOOD DRYING PROCESS ${ }^{1}$
}

\author{
R. ČIEGIS ${ }^{1}$ and V. STARIKOVIČIUS ${ }^{2}$ \\ ${ }^{1}$ Vilnius Gediminas Technical University \\ Saulètekio al. 11, LT-2040 Vilnius, Lithuania \\ E-mail: rc@fm.vtu. It \\ ${ }^{2}$ Vilnius University, Faculty of Mathematics and Informatics \\ Naugarduko 24, LT-2600 Vilnius, Lithuania \\ E-mail: vs@sc.vtu. It
}

Received September 19, 2002

\begin{abstract}
This work focuses on the development of mathematical models describing moisture movement in wood, when the wood is considered as porous medium. Main moisture transport mechanisms are discussed. It is shown how the wood can be described as a two- or threephase system. Summaries of several multiphase flow models are presented in the hierarchical order: from the most general models to more simple examples. The approximation steps are described explicitly, and all assumptions are given in detail. It shown how models for specific applications in wood drying or saturation can be obtained.
\end{abstract}

Key words: wood drying, mathematical models, multiphase flow.

\section{INTRODUCTION}

Development of mathematical models to describe wood drying process has been a topic of scientific research for many decades [17]. Since Tuttle (1925) [22] have presented the first mathematical theory for wood drying, numerous models have been developed. Basically these models can be classified into three categories: empirical curve-fitting equations, moisture diffusion equations, and fundamental heat and mass transfer equations.

Historically, most models are based on the moisture diffusion equation, which is derived using Fick's law. They are widely used due to their relative

\footnotetext{
${ }^{1}$ This work was supported in part by Lithuanian State Science and Studies Foundation grant T-576 and Eureka grant OPTPAPER 2623, E-2002.02.27
} 
simplicity and for many applications proved to be satisfactorily accurate [4; $6 ; 18 ; 22]$. The use of diffusion equation is usually restricted to the drying below the fiber saturation point (FSP), where only water vapor and bound water are involved and transported by molecular diffusion. However, there is precedent and some rationale for using the framework of the mathematics of diffusion for low-intensity wood drying even above the FSP [7; 12]. In this case, an assumption is made that the drying rate is proportional to the moisture gradient in the wood. No assumptions are made about mechanisms of moisture movement in the wood, which are not considered in such models. The coefficient of diffusion equation is called an internal moisture transfer coefficient instead of diffusion coefficient. Several methods are developed to determine the internal and surface moisture transfer coefficients [7;18].

However, over the last decades the development of more accurate and general models $[15 ; 16]$ becomes more popular. In such models the wood is considered as porous medium and multiphase flow and heat transfer is taken into account. These models are derived from fundamental mathematical models describing multiphase flow and heat transfer phenomena in porous media, which are based on principles of conservation. Since such models allow more accurate modeling in general, the use of them becomes inevitable for some applications. For example, high-intensity vacuum drying of wood cannot be modeled by models based on the diffusion equation.

This paper focuses on the development of mathematical models describing moisture movement in the wood, when the wood is considered as porous medium. First in Section 2, we discuss the main moisture transport mechanisms and show how the wood can be described as two- or three-phase system. In Section 3, we review fundamental multiphase flow and heat transfer models. Summaries of several models are presented in hierarchical order: from the most general to more simple and approximated ones. In Section 4, we give an example how models for specific applications can be derived from these basic models. Finally, conclusions are made in Section 5.

\section{MECHANISMS OF MOISTURE MOVEMENT IN WOOD}

In this section we will describe main mechanisms, which are important for moisture movement in wood.

Moisture exists in the wood as a bound water within the walls of the cells, free water in liquid form and water vapor in gas form in the voids of wood. The main difficulty in modeling moisture motion in wood is the fact that more than one mechanism may contribute to the total flow of water and the relative contribution of different mechanisms may change as the drying process proceeds. Modeling is also complicated because wood is an anisotropic medium.

If in the permeable wood the moisture content (MC) is above the FSP, then the existing free water can be transported through the interconnected voids by hydrodynamic flow due to the pressure difference or driving force, 
like gravity. The pressure difference in liquid phase is caused mainly due to the capillarity - molecular attraction between the liquid and the solid matrix, but it also can be the connected to external pressure (e.g. vacuum), thermal expansion, internal steam pressure.

At temperatures approaching and exceeding the boiling point of water, rapid vapor generation may produce significant total pressure gradients in addition to partial vapor pressure gradients. Water vapor will be moved from a high pressure to a low pressure regions by the gas-dynamic flow under total pressure difference. Although the density of water vapor is small, the volume flow rate is large under the certain pressure differences and significant amount of moisture may be transferred by water vapor gas-dynamic flow.

As an example we consider the vacuum drying. In vacuum drying [15], the lumber is placed in a tight drying chamber. The vacuum drying system pulls a vacuum on the lumber so that the water in wood is boiling and is drawn out of the wood. The gas-dynamic flow of water vapor becomes a dominant transport mechanism in moisture movement. Vacuum drying is actually based on the fact that the boiling point of water is substantially lowered when the atmospheric pressure over the wood is lowered. Usually, it is about $40 \mathrm{C}$.

In cyclic vacuum drying, the lumber is also in addition intermittently heated by a hot air convection. After the wood has reached the required temperature, a vacuum is pulled on the lumber. The drying starts and takes place till the drying rate is very small, then the whole cycle is repeated.

Unlike bulk flows of free liquid and vapor, diffusion process is the transfer of a material by essentially random molecular motion of single molecules in response to concentration gradients. Water and other small molecules can migrate across cell walls even when wood is impermeable and there is no pressure gradient.

In Fickian diffusion, all water molecules are free to migrate. They generally diffuse from a region of high moisture content to a region of low moisture content, which reduces the moisture gradient and equalizes the moisture content. The rate of diffusion is increasing with temperature. This is the principle reason for drying the wood at higher temperatures. Diffusion plays an important role in the drying of lumber, at all moisture content with impermeable wood and in permeable wood wherever the moisture content is too low for hydrodynamic flow of free water and there is no total pressure difference for gas-dynamic flow of water vapor.

There are two types of diffusion occurring in the wood, i.e. vapor diffusion and bound water diffusion. Vapor diffusion is the transfer of water vapor through the air in the voids of the cells. Bound water diffusion is water transfer within the walls of the cells.

In the Figure 1, we show how the wood is described as a three-phase system. The moisture phases and main transport mechanisms are presented. The phase changes include evaporation and condensation, absorption and desorption. 


$\begin{array}{ccc}\swarrow & \text { Moisture in wood } & \\ \text { Free Water } & \downarrow & \searrow \\ \text { in voids of cells } & \text { in voids of cells } & \text { Bound Water } \\ \downarrow & \downarrow & \downarrow \\ \text { liquid phase } & \text { gas phase } & \text { solid phase } \\ \downarrow & \downarrow & \downarrow \\ \downarrow & \text { Transport } & \downarrow \\ \text { 1) if }(M C>F S P & 2) \text { if permeable } & \text { 4) diffusion } \\ \text { and permeable }) & \text { gas-dynamic flow } & \text { within walls } \\ \text { hydrodynamic flow } & \text { 3) diffusion } & \\ & & \end{array}$

Figure 1. Wood as three-phase system.

\section{FUNDAMENTAL MULTIPHASE FLOW MODELS}

The multiphase flow in porous media has gained recently a lot of attention. This is due to the fact that problems involving the multiphase flow, heat transfer, and multicomponent mass transport in porous media arise in a broad spectrum of engineering disciplines. Important technological applications include the drying of porous solids and soils [27], subsurface contamination and remediation [2], thermally enhanced oil recovery, geothermal energy production, porous heat pipes [23], multiphase trickle bed reactors, nuclear reactor safety analysis, high-level radioactive waste repositories, paper machines.

The earliest modeling efforts involving multiphase flow in porous media began in the petroleum industry. Over the last decades a lot of efforts was made to create fundamental mathematical models for those phenomena. In this section, we review several fundamental multiphase flow and heat transfer models. We compile them in hierarchical order from the most general to more approximated and simplified in order to provide a basis for further model developments and applications to specific problems, as we will do in Section 4 for moisture movement in wood.

However, this is not an extensive review of the literature. The extensive reviews of multiphase flow and heat transfer studies are given in $[13 ; 14 ; 25$; 27].

\subsection{Multiphase Flow Model (MFM)}

Traditionally multiphase flow in porous media has been approached by socalled Multiphase Flow Model [2; 5], in which various phases are considered 
as distinct fluids with individual thermodynamic and transport properties and with different flow velocities. A brief summary of the MFM for multiphase, multicomponent transport in a porous medium is given below. The following set of governing macroscopic equations can be either semi-empirically postulated [5] or derived by the volume averaging method [2].

In latter case, the macroscopic equations are obtained by taking the average of the microscopic equations over the representative elementary volume (control volume). The microscopic equations are the equations of momentum, mass and energy conservation in all considered phases and at the interfaces. The control volume should be larger than the maximum dimension of any phase element, but much smaller than the characteristic scale of the system (Figure 2). Such scale changing enables to convert the real discontinuous medium to a fictitious continuous equivalent one. Each macroscopic term is obtained by averaging microscopic one. The averaging (integration) is done by using various closing assumptions (homogeneity, periodic cell structure etc.) [27].

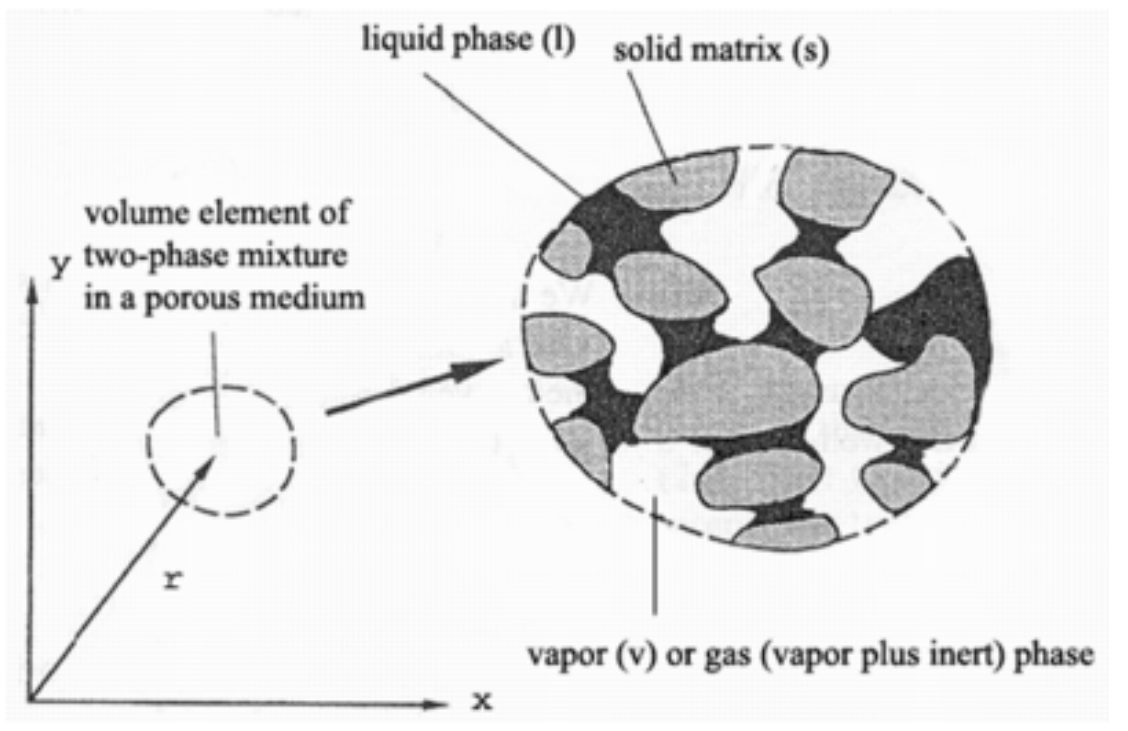

Figure 2. Schematic illustration of a control volume in multiphase system.

Here are the governing equations of MFM for multiphase, multicomponent transport in a non-deformable porous medium:

1. Mass conservation in phase $k$ :

$$
\varepsilon \frac{\partial\left(\rho_{k} s_{k}\right)}{\partial t}+\nabla \cdot\left(\rho_{k} \mathbf{u}_{k}\right)=\bar{m}_{k}
$$

where $\varepsilon$ is the porosity of porous medium, $\rho_{k}$ is the phase density, $s_{k}$ is the phase saturation denoting the volumetric fraction of the void space occupied 
by phase $k$,

$$
\sum_{k} s_{k}=1
$$

and $\mathbf{u}_{k}$ is the superficial (or Darcian) velocity vector (volume rate of flow through a unit cross-sectional area of multiple fluids and porous medium). It is also called bulk velocity. The term $\bar{m}_{k}$ represents an interfacial mass transfer rate from all other phases to phase $k$. In the absence of any external mass source or sink, it follows that

$$
\sum_{k} \bar{m}_{k}=0
$$

2. Momentum conservation in phase $k$ :

$$
\mathbf{u}_{k}=-\mathbf{K} \frac{k_{r k}}{\mu_{k}}\left(\nabla p_{k}-\rho_{k} \mathbf{g}\right),
$$

where $\mathbf{K}$ is the absolute permeability tensor of porous medium, $k_{r k}$ is the relative permeability of phase $k, \mu_{k}$ is the phase dynamic viscosity, $p_{k}$ is the phase pressure, and $\mathbf{g}$ is the acceleration vector due to gravity. The difference between the pressures for two adjacent phases $k$ and $j$ is called a capillary pressure $\left(p_{c k j}=p_{k}-p_{j}\right)$. It is related to saturations, pore size, pore shape, the angles at which fluid water air interface contacts the solid surface, the density difference between phases, temperature and radii of the curvature of the interface.

This generalized Darcy's law is valid if inertia as well as (macroscopic) viscous, boundary effects can be neglected, for example if velocities of fluids are quite small. In other case, well-established expressions from analogy with Navier-Stokes equation should be used [3; 13]. For example, a quadratic inertia term is often introduced for modeling the inertia effect.

3. Mass conservation of component $\alpha$ in phase $k$ :

$$
\frac{\partial}{\partial t}\left(\varepsilon \rho_{k} s_{k} C_{k}^{\alpha}\right)+\nabla \cdot\left(\rho_{k} \mathbf{u}_{k} C_{k}^{\alpha}\right)=-\nabla \cdot \mathbf{j}_{k}^{\alpha}+\bar{J}_{k}^{\alpha},
$$

where $C_{k}^{\alpha}$ is the mass concentration of component (species) $\alpha$ in phase $k$,

$$
\sum_{\alpha} C_{k}^{\alpha}=1
$$

and $\mathbf{j}_{k}^{\alpha}$ is an average flux vector which represents the nonadvective (diffusive) flux of component $\alpha$ in phase $k$ due to molecular diffusion and/or hydrodynamic dispersion. It is usually expressed in Fickian form:

$$
\mathbf{j}_{k}^{\alpha}=-\varepsilon \rho_{k} s_{k} \mathbf{D}_{k}^{\alpha} \nabla C_{k}^{\alpha},
$$


where $\mathbf{D}_{k}^{\alpha}$ is a macroscopic second-order tensor incorporating both diffusive and dispersive effects. The last term $\bar{J}_{k}^{\alpha}$ in equation (3.5) denotes the interface transfer rate of component $\alpha$ caused by chemical reaction (chemical nonequilibrium) and/or phase change at the interfaces between phase $k$ and all other phases. Recognizing that production of component $\alpha$ in phase $k$ must be accompanied by destruction of component $\alpha$ in other phases, it follows that

$$
\sum_{k} \bar{J}_{k}^{\alpha}=0
$$

It is assumed that there is no external generation of components due to the chemical or biological reactions.

4. Energy conservation in phase $k$ :

$$
\frac{\partial}{\partial t}\left(\varepsilon \rho_{k} s_{k} h_{k}\right)+\nabla \cdot\left(\rho_{k} \mathbf{u}_{k} h_{k}\right)=\nabla \cdot\left(\varepsilon s_{k} k_{k} \nabla T\right)+\bar{q}_{k},
$$

where local thermal equilibrium among phases has been assumed $\left(T_{k}=T\right.$ for all $k$ ), and $k_{k}$ and $\bar{q}_{k}$ represent the effective thermal conductivity of phase $k$ and interphase heat transfer rate associated with phase $k$, respectively. Hence,

$$
\sum_{k} \bar{q}_{k}=q
$$

where $q$ is an external volumetric heat source or sink. The phase enthalpy $h_{k}$ is related to common temperature $T$ via

$$
h_{k}=\int_{0}^{T} c_{k} d T+h_{k}^{0}
$$

where $c_{k}$ and $h_{k}^{0}$ represent the effective specific heat and the reference enthalpy of phase $k$, respectively. The validity of the local thermal equilibrium assumption is discussed in $[3 ; 13]$.

The above basic conservation laws provide a system of governing equations for the unknown vector velocities $\mathbf{u}_{k}$, scalar pressures $p_{k}$, scalar saturations $s_{k}$, mass concentrations $C_{k}^{\alpha}$, and the common temperature T. In general case, the averaging can be seen as a process of giving the guidance: what kind of terms must be included into macroscopic equations. However, it cannot eliminate completely the need in the postulation. To close the system, we need an additional set of equations.

The constitutive relationships specify how the phases interact with themselves, with each other and with porous medium. The state equations specify the thermodynamic state of the phases as functions of those state variables that determine it. The closure conditions are determined by experiments. 
Commonly, these experiments are made for very specific flows and flow situations and may be often valid only for specific application in a narrow range of flow conditions and concentrations.

The availability and reliability of constitutive relationships for moisture movement in wood are the main difficulties in multiphase modeling approach. However, there is a scientific literature devoted to capillary pressure [19], relative permeability [20], etc.

In the multiphase flow models, an assumption is commonly made that the gas (vapor) phase is ideal from thermodynamic point of view. Then modified state equations for an ideal gas are often used to determine the temperature in isothermal two-phase zone [23] or to express the gas phase density as a function of temperature and pressure.

Solving practical problems with MFM, which usually involve multi-dimensional effects, gravity, capillarity and phase change, requires a solution of multiply coupled sets of nonlinear differential equations. For this reason, there has been a great number of studies to develop robust numerical algorithms [10] for MFM on the one side and to derive approximate, simplified models [14] on the other. One of such models is recently developed multiphase mixture model [24].

\subsection{Multiphase Mixture Model (MMM)}

A key idea in the multiphase mixture model is a concept of the multiphase mixture, which is considered as a single fluid consisting of diffusing constituents (phases). Multiphase flow is then described in terms of mixture properties and characteristics (density, velocity and etc.) by set of conservation equations, which are derived from classical MFM. Also, explicit relations describing the relative motions between the multiphase mixture and individual phase are obtained and can be used to determine the individual phase variables from the mixture variables. Therefore, no phase characteristics are lost.

Here are the basic definitions of the MMM. The mixture density, velocity, species $\alpha$ concentration and enthalpy are defined, respectively, as

$$
\rho=\sum_{k} \rho_{k} s_{k}, \rho \mathbf{u}=\sum_{k} \rho_{k} \mathbf{u}_{k}, \rho C^{\alpha}=\sum_{k} \rho_{k} s_{k} C_{k}^{\alpha}, \rho h=\sum_{k} \rho_{k} s_{k} h_{k} .
$$

The mixture kinematic viscosity and the mobility of phase $k$ (sometimes called the fractional flow function) are given by

$$
\nu=\left(\sum_{k} \frac{k_{r k}}{\nu_{k}}\right)^{-1}, \quad \lambda_{k}=\frac{k_{r k}}{\nu_{k}} \nu, \quad \sum_{k} \lambda_{k}=1 .
$$

The mixture pressure is defined in the model so that the following differential equation holds:

$$
\nabla p=\sum_{j} \lambda_{j} \nabla p_{j}=\nabla p_{k}+\sum_{j} \lambda_{j} \nabla p_{c j k}
$$


Note that such pressure definition implies restrictions on the constitutive relationships like relative permeabilities and capillary pressure functions, and so on the applicability of MMM.

Here is the summary of governing equations of MMM:

1. Mass conservation of the multiphase mixture:

$$
\varepsilon \frac{\partial \rho}{\partial t}+\nabla \cdot(\rho \mathbf{u})=0
$$

2. Momentum conservation of the multiphase mixture:

$$
\rho \mathbf{u}=-\frac{\mathbf{K}}{\nu}\left(\nabla p-\left(\sum_{k} \lambda_{k} \rho_{k}\right) \mathbf{g}\right)=-\frac{\mathbf{K}}{\nu}\left(\nabla p-\gamma_{\rho} \rho \mathbf{g}\right),
$$

where $\gamma_{\rho}$ is called the density correction factor. Similarly to MFM, modified momentum conservation equation from analogy with Navier-Stokes equation are used to include the non-Darcian effects into multiphase mixture model [26].

The phase velocity $u_{k}$ is expressed as

$$
\rho_{k} \mathbf{u}_{k}=\mathbf{j}_{k}+\lambda_{k} \rho \mathbf{u},
$$

where $\mathbf{j}_{k}$ is the diffusive mass flux of phase $k$ within the multiphase mixture and it can be expressed as follows:

$$
\mathbf{j}_{k}=\frac{\mathbf{K} \lambda_{k}}{\nu} \sum_{i} \lambda_{i} \nabla p_{c i k}+\frac{\mathbf{K} \lambda_{k}}{\nu} \sum_{i} \lambda_{i}\left(\rho_{k}-\rho_{i}\right) \mathbf{g} .
$$

3. Mass conservation of component $\alpha$ in the multiphase mixture:

$$
\begin{aligned}
\varepsilon \frac{\partial}{\partial t}\left(\rho C^{\alpha}\right) & +\nabla \cdot\left(\gamma_{c}^{\alpha} \rho \mathbf{u} C^{\alpha}\right)=\nabla \cdot\left(\varepsilon \rho \mathbf{D}^{\alpha} \nabla C^{\alpha}\right) \\
& +\nabla \cdot\left(\varepsilon \sum_{k}\left(\rho_{k} s_{k} \mathbf{D}_{k}^{\alpha}\left(\nabla C_{k}^{\alpha}-\nabla C^{\alpha}\right)\right)\right)-\nabla \cdot\left(\sum_{k} C_{k}^{\alpha} \mathbf{j}_{k}\right)
\end{aligned}
$$

where $\gamma_{c}^{\alpha}$ is a correction factor for species advection and $\mathbf{D}^{\alpha}$ is the effective diffusion coefficient for the multiphase mixture. They are defined respectively as

$$
\gamma_{c}^{\alpha}=\frac{\rho \sum_{k} \lambda_{k} C_{k}^{\alpha}}{\sum_{k} \rho_{k} s_{k} C_{k}^{\alpha}}, \quad \rho \mathbf{D}^{\alpha}=\sum_{k} \rho_{k} s_{k} \mathbf{D}_{k}^{\alpha} .
$$

4. Energy conservation for the multiphase mixture:

$$
\frac{\partial}{\partial t}\left((1-\varepsilon) \rho_{s} h_{s}+\varepsilon \rho h\right)+\nabla \cdot\left(\gamma_{h} \rho \mathbf{u} h\right)=\nabla \cdot\left(k_{e f f} \nabla T\right)-\nabla \cdot\left(\sum_{k}\left(h_{k} \mathbf{j}_{k}\right)\right)+q
$$


where $k_{e f f}$ is an effective thermal conductivity of the composite system consisting of the solid matrix and the multiphase mixture, $\gamma_{h}$ is a correction factor for energy advection, $q$ is an external volumetric heat source or sink.

An advantage of MMM is that it requires much fewer nonlinear and coupled differential equations to be solved comparing with MFM. It avoids also such difficulties as the presence of the moving and irregular interfaces between the single- and multiphase regions. The model is single-domain formulation and all governing equations are valid throughout a whole problem domain including single- and multiphase regions. In addition, the formulation strongly resembles the single-phase transport theory and, thus, is amenable to solutions by conventional numerical algorithms.

The MMM approach allows also to obtain several other models for some simplified conditions. For two-phase single-component systems (liquid and vapor), the following liquid saturation equation can be obtained by substituting expression (3.17) into equation (3.1)

$$
\varepsilon \frac{\partial\left(\rho_{l} s_{l}\right)}{\partial t}+\nabla \cdot\left(\rho \mathbf{u} \lambda_{l}\right)=-\nabla \cdot \mathbf{j}_{l}+\bar{m}_{l}
$$

and used in conjunction with the mixture continuity equation (3.15) and Darcy law (3.16). Further simplifications can be made for immiscible, incompressible fluids.

\subsection{Unsaturated Flow Theory (UFT)}

In order to alleviate the analytical and numerical difficulties associated with MFM, an approximate model called the Unsaturated Flow Theory was developed and widely used by hydrologists [14]. Applications of UFT to the other multiphase problems in porous media were also made. In this subsection, we briefly derive from MFM the well-known Richards equation of UFT to gain insight into the important assumptions made in the theory and thus identify its limitations in certain situations.

Substituting equation (3.4) into equation (3.1) for a liquid phase (e.g., water in hydrology), we obtain

$$
\varepsilon \frac{\partial\left(\rho_{l} s_{l}\right)}{\partial t}+\nabla \cdot\left(-\mathbf{K} \frac{k_{r l}}{\nu_{l}} \nabla p_{l}+\mathbf{K} \frac{k_{r l}}{\nu_{l}} \rho_{l} \mathbf{g}\right)=\bar{m}_{l} .
$$

This provides the single equation for two unknowns, $s_{l}$ and $p_{l}$. In order to close the model, it is traditionally assumed in UFT that the gas phase (e.g., air in hydrology) essentially remains at the constant pressure (e.g., equal to atmospheric pressure):

$$
p_{g}=\text { const } \text {. }
$$

From the capillary pressure definition, it follows then that $\nabla p_{l}$ is simply given by

$$
\nabla p_{l}=\nabla p_{g}-\nabla p_{c}=-\frac{d p_{c}}{d s_{l}} \nabla s_{l} .
$$


Using equation (3.25) and neglecting the interphase mass transfer $\bar{m}_{l}$, we obtain from equation (3.23) the well-known Richards equation:

$$
\varepsilon \frac{\partial\left(\rho_{l} s_{l}\right)}{\partial t}=\nabla \cdot\left(\mathbf{D} \nabla s_{l}\right)-\nabla \cdot\left(\mathbf{K} \frac{k_{r l}}{\nu_{l}} \rho_{l} \mathbf{g}\right)
$$

where the diffusion coefficient is defined as

$$
\mathbf{D}\left(s_{l}\right)=-\mathbf{K} \frac{k_{r l}}{\nu_{l}} \frac{d p_{c}}{d s_{l}} .
$$

So, the main limitations of UFT are that it neglects the gas phase motion, interphase mass transfer, and effects of heterogeneity on the capillary pressure.

\section{OTHER MODELS FOR MOISTURE MOTION IN WOOD}

In previous sections we have showed how the general MFM, MMM, UFT, and other multiphase models can be applied to simulate the processes of moisture motion in the wood. The presented hierarchy provides us with important limitations of the models for specific applications. We note that Richards equation resembles classical moisture diffusion equation. However because of (3.24), it can be applied only in low-intensity conditions of conventional drying, when gas dynamics can be neglected. The use of multiphase models is inevitable in high-intensity drying conditions, for example, in vacuum drying, when the gas phase dynamics become critical.

In some applications, it is clear which process dominates the moisture transport in the wood and then the other processes can be neglected without the significant loss of accuracy. Then a simplified model can be obtained and used. In this section, we will obtain from MFM one differential model for modeling the conventional drying above the FSP or saturation of wood with chemical substances, when the first group processes dominate, and for conventional drying below FSP, when the third group processes dominate (see Figure 1).

In the first case we can apply assumption (3.24). From equation (3.23), we get for liquid phase:

$$
\frac{\partial c}{\partial t}+\nabla \cdot(\nu c)=\nabla \cdot(\mathbf{D} \nabla c)+\bar{m}_{l}
$$

where we define the moisture content $c(x, t)$ in the void of wood cell (there is almost no vapor in gas phase), diffusion $\mathbf{D}$ and convection $\nu$ coefficients, respectively, as

$$
c(x, t)=\varepsilon \rho_{l} s_{l}, \quad \mathbf{D}=-\mathbf{K} \frac{k_{r l}}{\varepsilon \mu_{l}} \frac{d p_{c}}{d s_{l}}, \quad \nu=\mathbf{K} \frac{k_{r l}}{\varepsilon s_{l} \nu_{l}} \mathbf{g} .
$$


If third group processes dominate (conventional drying below FSP and almost no free water left), then we have equation (3.5) for the vapor concentration $C_{g}^{w}$ in gas phase:

$$
\begin{aligned}
& \frac{\partial}{\partial t}\left(\varepsilon \rho_{g} s_{g} C_{g}^{w}\right)+\nabla \cdot\left(\rho_{g} \mathbf{u}_{g} C_{g}^{w}\right)=\nabla \cdot\left(\varepsilon s_{g} \rho_{g} \mathbf{D}_{g}^{w} \nabla C_{g}^{w}\right)+\bar{m}_{g} \\
& \varepsilon s_{g} \rho_{g} C_{g}^{w}=c(x, t), \quad s_{g}=1, \quad \mathbf{D}=\mathbf{D}_{g}^{w}, \quad \nu=\frac{1}{\varepsilon} \mathbf{u}_{g}, \\
& \frac{\partial c}{\partial t}+\nabla \cdot(\nu c)=\nabla \cdot(\mathbf{D} \nabla c)+\bar{m}_{g} .
\end{aligned}
$$

In both cases, we are not neglecting the interphase mass transfer with solid phase (absorption and desorption). For the bound water concentration $C_{s}^{w}$ in solid phase, neglecting all motion in the wood matrix, we get

$$
\begin{aligned}
& \frac{\partial}{\partial t}\left((1-\varepsilon) \rho_{s} C_{s}^{w}\right)+0=0+\bar{m}_{s}, \\
& (1-\varepsilon) \rho_{s} C_{s}^{w}=s(x, t), \\
& \frac{\partial s}{\partial t}=\bar{m}_{s},
\end{aligned}
$$

where $s(x, t)$ is moisture content in the wall of the cell.

It follows from equation (3.3) that

$$
\bar{m}_{l}+\bar{m}_{s}=0, \quad \bar{m}_{g}+\bar{m}_{s}=0 .
$$

In both cases, we couple the equations in the following way:

$$
\begin{aligned}
\frac{\partial c}{\partial t}+\nabla \cdot(\nu c) & =\nabla \cdot(\mathbf{D} \nabla c)-p(l(c) c-g(s) s) \\
\frac{\partial s}{\partial t} & =p(l(c) c-g(s) s)
\end{aligned}
$$

where nonnegative functions $l(c)$ and $g(s)$ describe moisture absorption and desorption and depend on pressure of vapor, relative humidity, chemical potential and moisture content in corresponding environment. Examples are constants $l(c)=l>0, g(s)=1$ and Henry law: $l(c)=l, g(s)=k /(1-\gamma s)$, where $\gamma>0$ and $\gamma s<1$, for any s.

The obtained differential model (4.1)-(4.2) is not new in its form and describes a broad class of real world problems. For example, similar systems were used for modeling the gas absorption by porous material [21] (without diffusion term), saturation of wood with certain chemical substances [1], and even the transport of soluble substances in rivers [11].

However, such model development has clear benefits even when formally not a new model is obtained. It allows to spot unknown or missed restrictions and 
limitations in the model and gives a vital information about the coefficients, which should be used. For example, modeling conventional drying above the FSP, we see, that diffusion coefficient $\mathbf{D}$ has nothing to do with the physical process of moisture diffusion in the wood.

This model was numerically investigated in our previous works. The efficient finite difference scheme for solving one-dimensional linear case was presented in [9]. The unconditional stability estimates and convergence of the discrete solution are proved in the maximum norm for different types of boundary conditions. The locally one-dimensional splitting scheme was proposed for three-dimensional linear case in [8]. The unconditional stability and convergence in the maximum norm are proved for a problem with the Robin boundary conditions.

\section{CONCLUSIONS}

In this paper, hierarchy of several fundamental multiphase flow models has been compiled. It is shown how these models can be applied to the wood considering it as two- or three-phase system. A simplified model describing moisture motion in the wood is derived from basic multiphase flow model using assumptions specific for certain applications.

\section{REFERENCES}

[1] A. Aboltinš, A. Buikis, J. Cepitis, H. Kalis and A. Reinfelds. Diffusion and chemical attachment of substances with simple molecular structure in wood. In: Progress in Industrial mathematics at ECMI98. B. G. Teubner, Leipzig, 188 - 195, 1999.

[2] L.M. Abriola and G.F. Pinder. A multiphase approach to the modeling of porous media contamination by organic compounds 1. Equation development. Water Resour. Res., 21, $11-18,1985$.

[3] A. Amiri and K. Vafai. Analysis of dispersion effects and non-thermal equilibrium, nondarcian, variable porosity incompressible flow through porous media. Int. J. Heat Mass Transfer, 37, 939 - 954, 1994.

[4] R. Baronas, F. Ivanauskas, I. Juodeikienè and A. Kajalavičius. Modelling of moisture movement in wood during outdoor storage. Nonlinear Analysis: Modelling and Control, 6(2), $3-14,2001$.

[5] J. Bear. Dynamics of Fluids in Porous Media. Elsevier, New York, 1972.

[6] X. Bui, E. Choong and W. Rudd. Numerical methods for solving the equation for diffusion through wood during drying. Wood Science, 13(2), 1991.

[7] Y. Chen, E.T. Choong and D.W. Wetzel. A numerical analysis technique to evaluate the moisture dependent diffusion coefficient on moisture movement during drying. Wood Fiber Sci., 28(3), 338 - 345, 1996.

[8] R. Čiegis and V. Starikovičius. The finite difference scheme for $3 \mathrm{~d}$ mathematical modeling of wood drying process. Computational methods in applied mathematics, 1(2), 123 $-137,2001$.

[9] R. Čiegis and V. Starikovičius. The finite difference scheme for wood drying process. Mathematical modelling and analysis, 6(1), 48 - 57, 2001. 
[10] R.E. Ewing and H. Wang. A summary of numerical methods for time-dependent advection-dominated partial differential equations. Journal of Computational and Applied Mathematics, 128, 423 - 445, 2001.

[11] M. Hilden and G. Steinebach. ENO-discretization in MOL-applications: some examples in river hydraulics. Applied Numerical Mathematics, 28, $293-308,1998$.

[12] O.A. Hougen, H. McCauley and W.R. Marshal. Limitations of the diffusion equation in drying. Trans. Am. Inst. Chem. Eng., 36, $183-202,1940$.

[13] M. Kaviany. Principles of Heat Transfer in Porous Media. Springler-Verlag, Berlin, 1991.

[14] H.J. Morel-Seytoux. Two-phase flow in porous media. Adv. Hydrosci., 9, 119 - 202, 1973.

[15] P. Perre and S. Mosnier. Vacuum drying with radiative heating. Vacuum Drying of Wood 95, 1995.

[16] O.A. Plumb, G.A. Spolek and B. A. Olmstead. Heat and mass transfer in wood during drying. Int. J. Heat Mass Transfer, 28(9), 1669 - 1678, 1985.

[17] J. Salin. Modeling of wood drying. Drying Technology, 9(3), 775 - 793, 1991.

[18] W.T. Simpson and J.Y. Liu. An optimization technique to determine red oak surface and internal moisture transfer coefficients during drying. Wood and Fiber Science, 29, $312-318,1997$.

[19] G. Spolek and O.A. Plumb. Capillary pressure of woods. Wood Sci. Tech., 15(3), 100 $-109,1981$.

[20] F.O. Tesoro, E.T. Choong and O.K. Kimbler. Relative permeability and the gross pore structure of wood. Wood and Fiber, 6(3), 226 - 236, 1974.

[21] A. Tihonov and A. Samarskij. Equations of the Mathematical Physics. Nauka, Moscow, 1972. (in Russian)

[22] F. Tuttle. A mathematical theory of the drying of wood. Journal of the Franklin Institute, 200(5), $609-614,1925$.

[23] K.S. Udell. Heat transfer in porous media considering phase change and capillarity the heat pipe effect. Int. J. Heat Mass Transfer, 28, 485-495, 1985.

[24] C.Y. Wang and P. Cheng. A multiphase mixture model for multiphase, multicomponent transport in capillary porous media. Part I: Model development. Int. J. Heat Mass Transfer, 39, 3607 - 3618, 1996.

[25] C.Y. Wang and P. Cheng. Multiphase flow and heat transfer in porous media. Advances in Heat Transfer, 300, 93 - 196, 1997.

[26] Z.H. Wang, C.Y. Wang and K.S. Chen. Two-phase flow and transport in the air cathode of proton exchange membrane fuel cells. Journal of Power Sources, 4094, 1-11, 2000.

[27] S. Whitaker. Simultaneous heat, mass, and momentum transfer in porous media: A theory of drying. Advances in Heat Transfer, 13, $119-203,1977$.

\section{Medienos džiovinimo proceso matematiniai modeliai}

\section{R. Čiegis, V. Starikovičius}

Šiame straipsnyje pagrindinis dèmesys skiriamas drègmès koncentracijos kitimo medienoje matematiniam modeliavimui, kai mediena yra nagrinėjama kaip poringa terpè. Yra aptarti pagrindiniai skysčiu judejjimo mechanizmai, naudojant dvifazius ir trifazius artinius. Sudaryta hierarchinè modelių sistema, apimanti tiek pačius bendriausius modelius, tiek ir ju supaprastintus varijantus. Visi modelių išvedimo žingsniai aprašomi išreikštiniu būdu, griežtai suformuluotos tokių modeliu teisingumo prielaidos. Parodyta, kad esant tam tikroms sąlygoms iš šių modelių galime gauti gerai žinomus artinius, teoriškai pagrisstos jų koeficientu išraiškos. 